\title{
Progression of disease in HIV infected children slowed after the first year of life
}

\author{
The European Collaborative Study. Fluctuations in symptoms in human immunodeficiency virus-infected children: the first \\ 10 years of life. Pediatrics $2001 \mathrm{Jul} ; 108: 116-22$.
}

\section{QUESTION: In children with HIV infection at birth, who received differing treatment protocols, what are the clinical and immunological manifestations during the first 10 years of life?}

\section{Design}

Cohort study with a median of 5.8 years of follow up.

\section{Setting}

11 paediatric centres in 7 European countries.

\section{Patients}

$170 \mathrm{HIV}$ infected children who were born to mothers known to be infected with HIV at the time of delivery.

\section{Assessment of prognostic factors}

Children were categorised into 3 cohorts according to the treatment policy at the time of birth. Cohort 1 consisted of children born from 1985-8 when no recommendations for treatment existed; cohort 2 consisted of children born from 1989-94 when the treatment policy was restricted to monotherapy for symptomatic children; and cohort 3 consisted of children born from 1995-9 when the initiation of combination therapies was recommended at an early stage. Children were allocated to Centres for Disease Control and Prevention (CDC) categories for clinical manifestations ( $\mathrm{N}=$ asymptomatic, $\mathrm{A}=$ mildly symptomatic, $\mathrm{B}=$ moderately severe symptoms including lymphoid interstitial pneumonitis, $\mathrm{C}=$ severe symptoms, and $\mathrm{D}=$ death) and immunological classes $(1=$ normal immune suppression, $2=$ moder ate immune suppression, and $3=$ severe immune suppression) at each visit to the clinic.

\section{Main outcome measures}

Death and severity of disease progression with actual treatment received.

\section{Main results}

70 children $(41 \%)$ progressed to AIDS. 45 children (26\%) died of AIDS during follow up, and 2 children died of non-HIV related causes. 45 of 55 children (82\%) who never received antiretroviral treatment developed HIV related signs and symptoms. It was estimated that $>15 \%$ of infected children will have progressed to severe disease (category $\mathrm{C}$ ) or death (category $\mathrm{D}$ ) by 1 year of age, after which the rate would be $7 \%$ annually to just under $40 \%$ by 5 years and just under $50 \%$ by 10 years. Also, an estimated $50 \%$ of children would have progressed to moderate immune suppression (category 2) by 1 year of age and $>90 \%$ by 5 years. The children in all 3 cohorts differed in their rate of disease progression, and the children in cohort 3 were the least likely to progress to serious disease (category C). It was estimated that by 1 year of age, $>25 \%$ of cohort 1 would have progressed to severe disease (category C) compared with $15 \%$ in cohort 2 and only $5 \%$ in cohort $3(\mathrm{p}=0.011)$.

\section{Conclusions}

In children with HIV infection at birth, progression to clinical and immunological manifestations of severe disease slowed after the first year of life. Children were without serious symptoms or signs for most of the time.

\section{COMMENTARY} dren infected perinatally in 11 European centres since 1985. severity of infections; and developmental outcomes. ${ }^{1}$ staff is experienced in assessing and treating such children. outcomes of infants of mothers with HIV. Nurs Res 2001;50:5-14.
Sources of funding: Medical Research Council, UK and European Commission.

For correspondence: Professor M L Newell, Centre for Paediatric Epidemiology and Biostatistics, Institute of Child Health, 30 Guilford Street, London WCIN 1EH, UK.

A modified version of this abstract appears in

Evidence-Based

Medicine.

The European Collaborative Study is an important longitudinal study providing rich information about the clinical patterns and immunological progression of HIV in chil-

Analysis of such data is complicated because of the ever changing treatments for HIV, but the investigators analysed the effect of treatment changes in their study by dividing the sample into 3 groups according to treatment policy at the time of birth. Clinical status was measured using the CDC classification system at each visit. Nurses are interested in broader aspects of clinical response including symptom distress; number, type, and

Immune status did not reflect clinical stage at any age and progress of disease was difficult to predict by immune status. CD 4 count poorly reflects virological activity, which fluctuates because the count is affected by treatment. Currently, viral load is used as an indicator of disease progression. However, it may not be a good indicator in children $<3$ years of age. After 3 years of age, HIV1 RNA plateaus and is a more useful indicator. Assessment and follow up of young children with HIV should be done at centres whose

Of particular note from this study is that children with HIV may do better than we thought. The overall progression of disease slows after the first year of life, is less rapid from 1-5 years of age, and slows even more from 5-10 years. It will be increasingly important to provide support to children and families using models of chronic illness care rather than treating HIV as a terminal illness. Developmental outcomes, preventive health care, adherence to treatment, and maintaining contact with healthcare providers are all issues that should be closely monitored in HIV infected children. As the children reach school age, their parents will need help in telling them about their illness. Furthermore, healthcare professionals working with adolescents with HIV are challenged by how to help them manage complicated issues, including sexuality, as they live with HIV.

Margaret S Miles, RN, PhD Professor, School of Nursing University of North Carolina at Chapel Hill Chapel Hill, North Carolina, USA

1 Holditch-Davis D, Miles MS, Burchinal M, et al. Parental caregiving and developmental 